\title{
Interventional radiology treatments for iatrogenic severe bleeding during percutaneous coronary interventions
}

\author{
Piotr Pasecki ${ }^{1 A, B, D, E, F}$, Jerzy Narloch ${ }^{1 A, B, D, E, F}$, Konrad Tkaczewski ${ }^{2 A, D, F}$, Wojciech Wasek ${ }^{2 A, F}$, Piotr Ziecina ${ }^{1 E}$, Krzysztof Brzozowski ${ }^{1 E}$ \\ 'Department of Interventional Radiology, Military Institute of Medicine, Warsaw, Poland \\ 2Department of Interventional Cardiology, Military Institute of Medicine, Warsaw, Poland
}

\section{Abstract}

\begin{abstract}
Purpose: Interventional cardiology and interventional radiology are separate medical disciplines in which intra-arterial contrast media are used. Interventional cardiology has resigned from many types of treatment techniques that are now used and developed in the field of interventional radiology. In the event of iatrogenic bleeding during coronary interventions, there is an urgent need to use safe and efficient rescue procedures that are as efficient as cardiosurgery but use simpler treatment options. Serious perforations require immediate endovascular interventions. Medical history may reveal risk factors for artery perforation. Medicines, location of artery perforation, and extent of bleeding are directly associated with the prognosis. Most often, arterial perforations are due to inappropriate wire manipulation or use of oversized balloons or cutting balloons. Prolonged, artery-occluding balloon inflation, covered stent implantation, and embolisation with different agents are among the available treatment options for artery ruptures.
\end{abstract}

Material and methods: A retrospective analysis was carried out among selected patients with iatrogenic vascular complications during procedures involving either coronary or non-coronary arteries.

Results: Only representative cases were selected and presented in the patient subsection.

Conclusions: Artery perforation during cardiac catheterisation can lead to dire consequences. To manage this complication, clinicians need pre-established procedures, adequate resources, and knowledge. Interventional radiology can be used as a salvage therapy in such cases.

Key words: PCI, haemorrhage, interventional radiology.

\section{Introduction}

Interventional cardiology and interventional radiology are separate medical disciplines in which intra-arterial contrasts are used. Interventional cardiology, which focuses on the management of coronary syndromes, has resigned from many types of treatment techniques, e.g. embolisation, that are still used and developed in the field of interventional radiology. In the event of iatrogenic bleeding during coronary interventions, it is imperative to use safe and efficient rescue procedures that are as efficient as cardiosurgery but use simpler treatment options.
Serious perforations require an immediate endovascular intervention [1-3]. If a patient experiences serious bleeding during percutaneous coronary intervention, proper treatment and delayed rescue angioplasty may be hindered. The patient's medical history may reveal risk factors for artery perforation. Medications such as antiplatelet and novel anticoagulant (NOACs) drugs that affect haemostasis should be considered. The location of artery perforation constitutes one of the most important factors for further treatment. If any medications are taken by the patient, which affect the superficial arteries, such as the radial, brachial, or axillary arteries, there is a possibility

Correspondence address:

Jerzy Narloch, MD, Department of Interventional Radiology, Military Institute of Medicine, Szaserow 128, 01-141 Warsaw, Poland, phone: +48 261816689 ,

fax: +48 261816854 , e-mail: jerzy.narloch@gmail.com

Authors' contribution:

A Study design · B Data collection · C Statistical analysis · D Data interpretation · E Manuscript preparation · F Literature search · G Funds collection 
that compression techniques or devices may be required to stop bleeding. If damage is located within the subclavian artery or the brachiocephalic trunk, compression cannot be used and therefore other techniques including surgery or endovascular embolisation should be considered. An injury to the coronary artery is more dangerous because it may lead to massive pericardial effusion and acute cardiac tamponade [3] or other serious complications such as myocardial infarction or cardiac arrhythmias [4]. These situations are life-threatening, and treatment delays may lead to cardiac arrest and even death. Typically, artery perforations occur due to aggressive wiring, oversized balloons, cutting balloons, roto-ablation, and most often due to rupture of a heavily calcified atherosclerotic plaque that penetrates through or beyond the adventitia [1-8]. Artery ruptures can be managed with prolonged, artery-occluding balloon inflation, covered stent implantation, or specific for interventional radiology, embolisation with different agents such as microcoils, gelatine sponge, autologous coagulated blood, thrombin, fibrin glue, collagen, subcutaneous tissue, cyanoacrylate liquid glue, trisacryl gelatine microspheres, or polyvinyl alcohol particles.
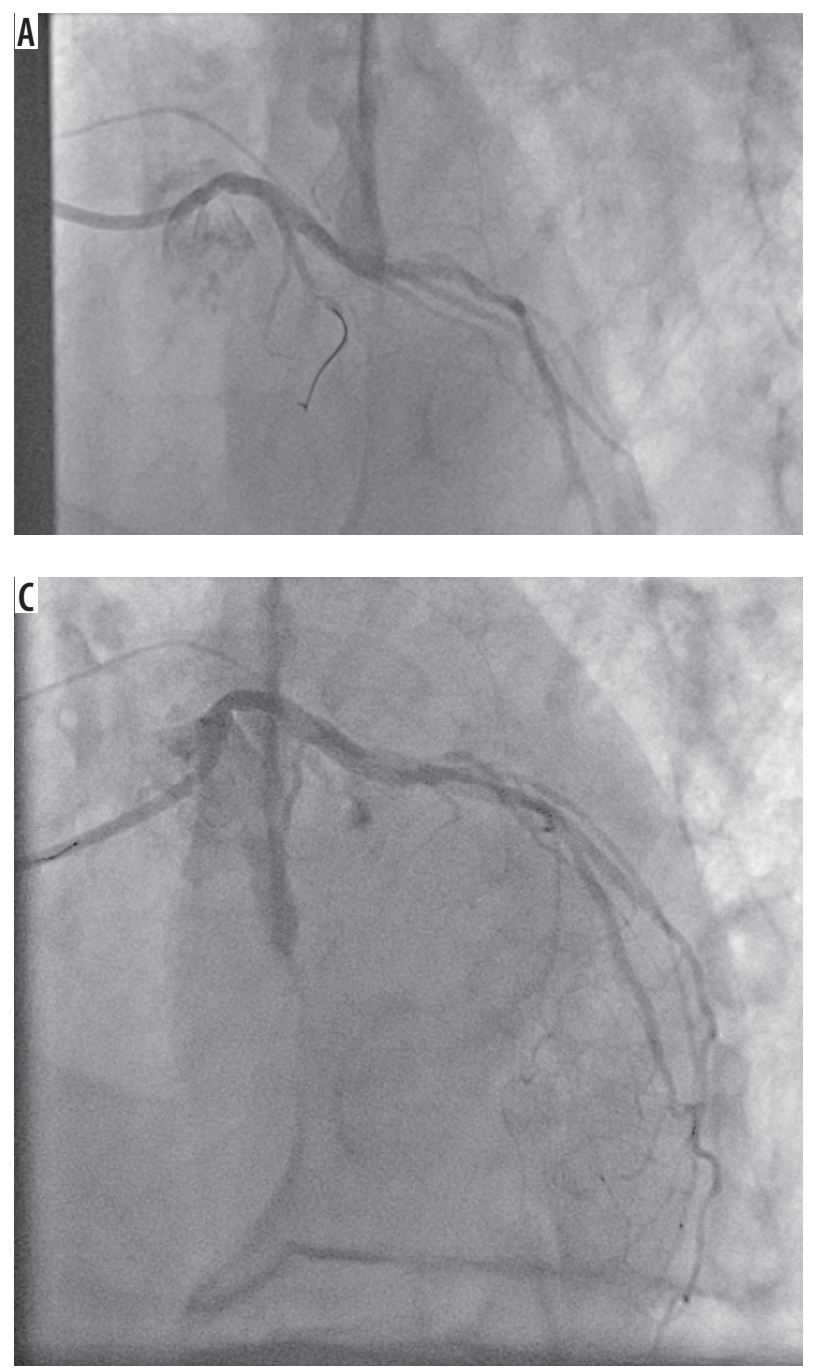

\section{Material and methods}

This study is a retrospective analysis of percutaneous coronary intervention (PCI) cases complicated by vessel rupture that occurred within a two-year period during 4000 diagnostic and 2000 therapeutic cardiac cauterisations that required interventional radiology techniques. Patients with bleeding complications within the afferent arteries of both upper and lower limbs, e.g. radial artery, were excluded from the analysis. Only representative cases were selected and are presented in the next subsection.

\section{Case 1}

A 70-year-old man with chronic coronary artery disease (CAD), after several myocardial infarctions (MI), treated with numerous percutaneous coronary interventions (PTCA), with post-myocardial infarction heart failure (HF), end-stage renal disease (ESRD), bladder cancer, and generalised atherosclerosis was admitted to the Intensive Care Unit of the Cardiology Department of our Institute with a diagnosis of acute coronary syndrome. He had a history of recurrent chest pain accompanied

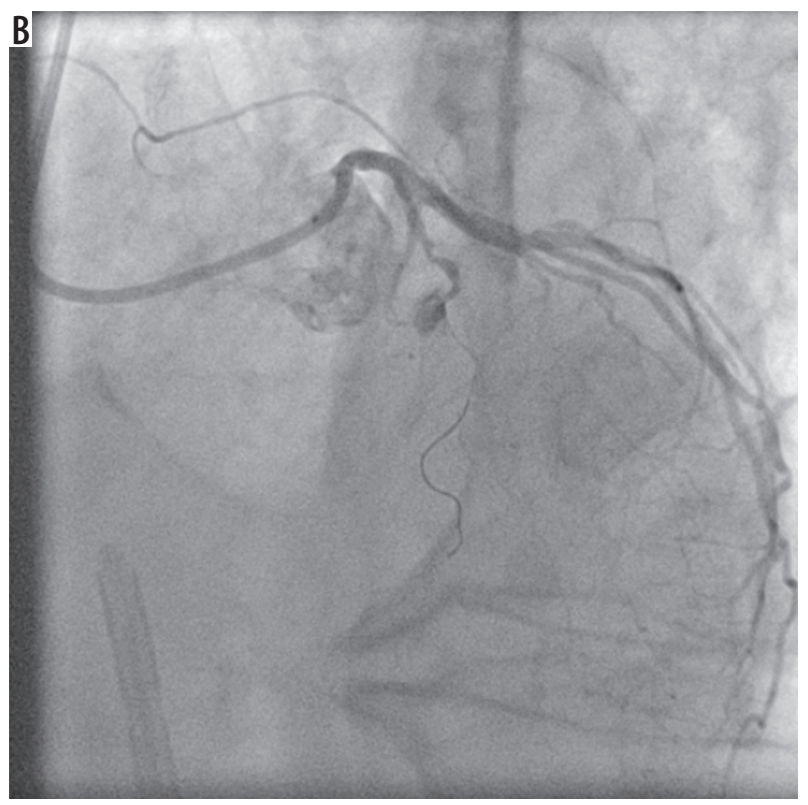

Figure 1. After opening left anterior descending (A), a balloon was placed in the origin of the first diagonal branch (D1), and after predilatation (not shown) within normal pressure range, an injection of contrast showed extravasation into the pericardium (B). Due to continued bleeding, the artery was embolised with a mixture of cyanoacrylate with lipiodol (C) 
by shortness of breath. On the day of admission, in the morning, he complained of an intense shortness of breath. On admission, the patient was in fair condition, without chest pain; blood pressure was 160/95 $\mathrm{mmHg}$, and heart rate was $80 / \mathrm{min}$. Electrocardiography (ECG) showed sinus tachycardia at $100 / \mathrm{min}$, pathological Q waves in leads V2-V4, and concave ST-elevation in leads V2-V3. Increasing troponin values were observed $-99.8 \mathrm{ng} / \mathrm{l}$ on arrival and $648 \mathrm{ng} / \mathrm{l}$ on the second measurement. Urgent coronary angiography was performed through the right radial access using a standard diagnostic $5 \mathrm{~F}$ catheter. The angiography revealed a dominant right coronary artery (RCA) with diffuse atherosclerotic lesions that caused up to 50-60\% stenosis and occluded the posterior descending artery (PDA). Small atherosclerotic changes were observed in the left main coronary artery (LMCA) trunk and the circumflex artery (CX). The left anterior descending (LAD) artery was occluded at the proximal part with good collateral circulation that maintained blood flow to the central and peripheral sections. After opening the LAD, the balloon was placed at the origin of the first diagonal branch (D1), and after predilatation within a normal pressure range, a contrast injection showed blood extravasation into the
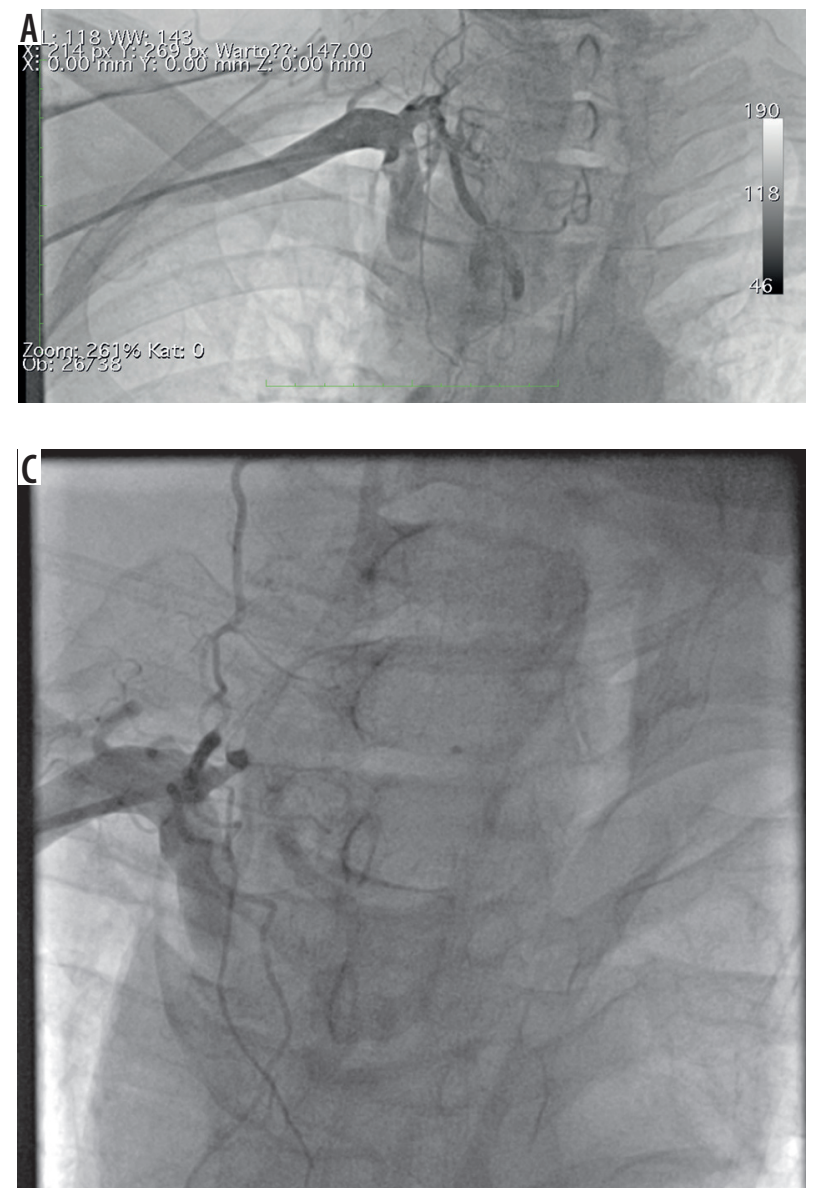

Figure 2. A) A guidewire was placed accidentally into the inferior thyroid artery, causing its perforation. B) To stop the bleeding, a balloon was placed and inflated proximally. () The procedure was insufficient, and embolisation with a mixture of cyanoacrylate with lipiodol was performed with success. D) Axial computed tomography scan showing a haematoma with contrast medium foci localised in the upper mediastinum and embolic material within the inferior thyroid artery branches
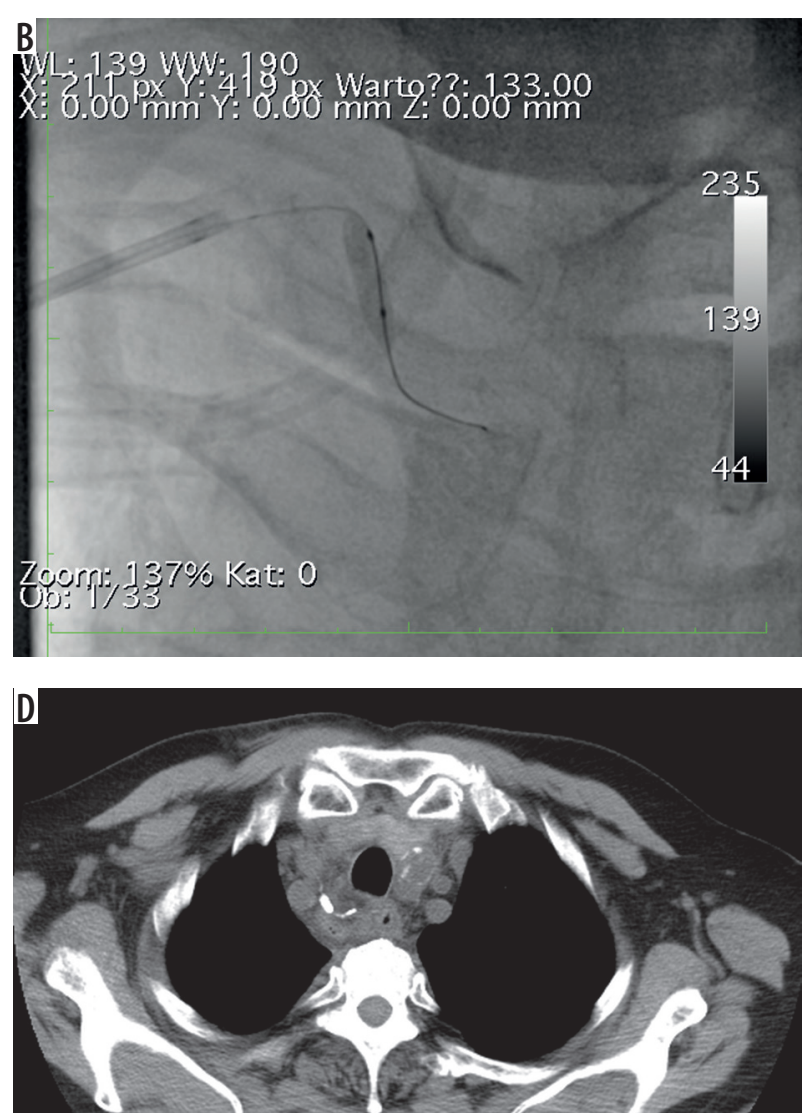

pericardium. The same balloon was immediately placed at that site and was kept inflated for at least three minutes with short breaks to control the effect. Due to continued bleeding, the interventional radiologist on duty was called to embolise the artery with a mixture of cyanoacrylate with lipiodol (1:2 ratio), which was performed with success (Figure 1). Echocardiography showed pericardial effusion of $9 \mathrm{~mm}$, with decreasing amounts of fluid on follow-up examinations. No additional intervention was needed. In the following days, the patient experienced an ischaemic stroke. Due to the recent LAD perforation, the decision was made not to administer thrombolysis. Although upon further observation the patient's clinical state improved, he developed sepsis and subsequent cardiac arrest; despite cardiopulmonary resuscitation (CPR), the patient died two weeks after admission.

\section{Case 2}

An 81-year-old man with a history of mild aortic valve stenosis and two ST-elevation myocardial infarctions (STEMI), in 2003 and 2004, treated with PCI with stent implantation in LAD and RCA was referred to our insti- 
tution due to unstable angina with transient chest pain. Cardiac symptoms started three days before hospitalisation. ECG showed ST segment depression in leads V3-V5, a biphasic $\mathrm{T}$ wave in lead V5, and an inversion in the V6 lead. Troponin T level was normal on two examinations. The patient was given $300 \mathrm{mg}$ of acetylsalicylic acid (ASA), $75 \mathrm{mg}$ of clopidogrel, and $60 \mathrm{mg}$ of enoxaparin SC for three days. Urgent coronarography was performed via the right radial approach on the day of admission. At the beginning of the procedure, a diagnostic 0.038 -inch wire followed by a guiding catheter was accidentally placed into the inferior thyroid artery, causing perforation of this artery with continuous bleeding into the upper mediastinum and neck, which was observed on angiography. Initially, the bleeding was observed and managed conservatively, and ceased spontaneously. After a proper catheter reposition, coronarography was performed, which showed critical LAD stenosis. Other coronary arteries were normal. Successful angioplasty of LAD was performed with stent implantation. A control contrast injection into the inferior thyroid artery showed persistent bleeding with an irregular shape formation - a presumed pseudoaneurysm. To stop the bleeding, a balloon was placed and inflated at the proximal segment of the injured artery. The procedure was unsuccessful and therefore an interventional radiolo-
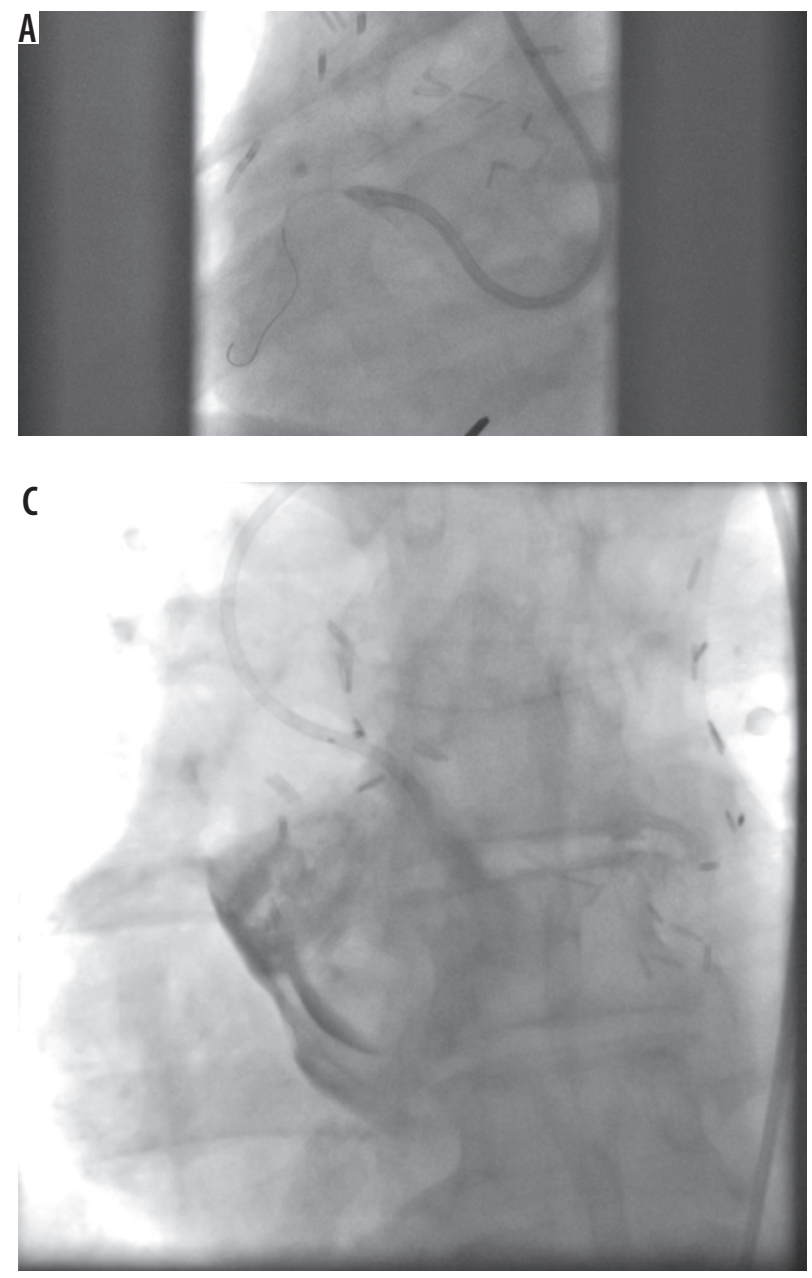

gist performed embolisation with a mixture of cyanoacrylate and lipiodol (1:2 ratio), with success (Figure 2). On computed tomography (CT), which was performed shortly after the procedure, a haematoma with approximate dimensions $34 \mathrm{~mm} \times 40 \mathrm{~mm} \times 45 \mathrm{~mm}$, with contrast medium foci in the upper mediastinum and embolic material within the inferior thyroid artery branches, was demonstrated. Additionally, smaller interstitial blood depositions on the right side of the neck were also observed. There were no signs of persistent bleeding on subsequent contrast-enhanced CT. The patient had no further recurrent cardiac symptoms. No clinical or laboratory signs of bleeding were noticed in the course of hospitalisation. The patient was discharged in good general condition.

\section{Case 3}

A 68-year-old man with a history of hypertension, CAD, $\mathrm{MI}$ in 2000, and coronary artery bypass grafting (CABG) in 2004 was admitted to the Cardiology Department for elective coronary catheterisation, which revealed multivessel CAD with patent left internal mammary artery (LIMA) bypass graft to LAD and totally occluded proximal RCA with origins of a saphenous vein graft (SVG) to RCA and SVG to obtuse marginal branch II (OM II).

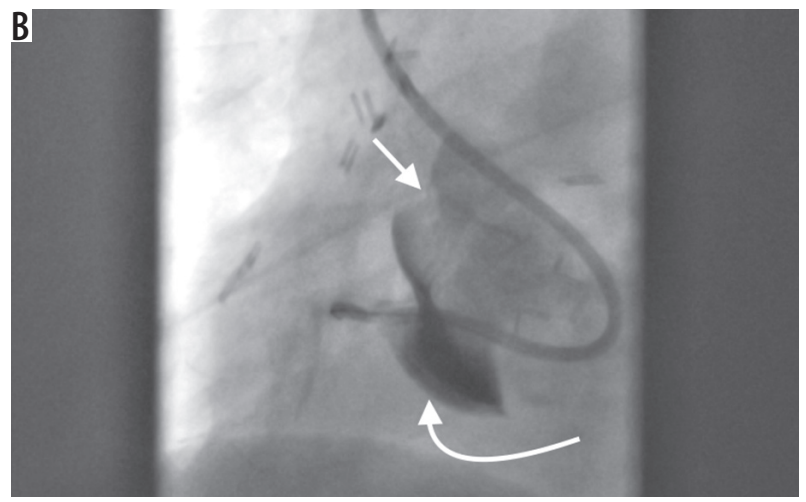

Figure 3. A) A guidewire was placed in a chronic totally occluded right coronary artery after multiple attempts with different guidewires. B) An angiogram showed an intramural haematoma (short arrow) with free dye extravasation into the medial layer of the aortic wall at the site of the right sinus of Valsalva (long arrow) (C) 
It was decided to proceed with PCI of the chronic totally occluded (CTO) RCA. Using an AL2, 6F guiding catheter, Pilot 50 and then Progress 80 wires were tried unsuccessfully to cross the RCA occlusion. An angiogram showed a large artery perforation with free dye extravasation into the medial layer of the aortic wall at the site of the right sinus of Valsalva (Figure 3). The patient remained well and haemodynamically stable. The line of aortic dissection did not propagate, and the patient was managed conservatively. At the coronary care unit (CCU), transthoracic echocardiography (TTE) showed no evidence of pericardial effusion or extension of the dissection. Transoesophageal echocardiography (TEE) strongly suggested an aortic intramural haematoma (IMH), and contrast-enhanced $\mathrm{CT}$ revealed a small hyperdense rim in that area with no clear dissection. The remaining course of hospitalisation was uneventful. Follow-up contrast-enhanced CT was performed three weeks later and showed no evidence of aortic dissection.

\section{Discussion}

Coronary perforations and serious bleeding complications occur rarely in interventional cardiology. Early diagnosis is based on imaging and clinical symptoms and signs, such as pain or resistance during guidewire advancement or an abrupt haemodynamic compromise, which is directly related to the magnitude of the perforation. Exceptional cases of spontaneous perforation of atherosclerotic vessels and traumatic perforations after diagnostic angiography have been described in the literature $[1,2,4-6,8]$. Most coronary perforations occur due to PTCA. Iatrogenic perforations are due to the use of sharp objects such as a guidewire, excessive balloon stretching, oversized balloons or stents, or mechanical disruption of the wall during atherectomy. Potential risk factors for arterial perforations, including coronary interventions, have been determined. The literature concerning this problem indicates that coronary artery perforation is infrequent $(0.3-$ $0.5 \%$ ) but linked to the use of specific devices during coronary interventions, especially in older and female patients with calcified lesions and tortuous or chronic totally occluded vessels. Perforations occur more frequently with plaque debulking than with non-debulking techniques [1,2]. The risk of perforation is ten times higher for mechanical atherectomy than for balloon angioplasty or guidewire use, which in turn are associated with a higher risk than stenting [1,2,4-6,8-11]. Antithrombotic treatment, especially concomitant use of GPIIb/IIIa inhibitors, can reveal an otherwise clinically silent vessel tear as a visible perforation [9]. Coronary perforations are classified according to their appearance on angiography into the three following types: type I - limited to the artery wall without blood extravasation, type II - pericardial or myocardial blushing, and type III - perforation $\geq 1 \mathrm{~mm}$ in size with contrast streaming and cavity spilling (some- times described as type IV) [10]. There are several management strategies depending on the coronary artery status, size of the perforation, extent of contrast extravasation, haemodynamic stability of the patient, and used medications. The artery should be sealed as soon as possible to prevent contrast extravasation into the pericardium, preventing cardiac tamponade. In general, prolonged balloon inflation at the site of perforation and anticoagulation reversal, if necessary, can be initial measures. In the meantime, a cardiac surgeon should be notified should an emergency surgery be required. There are several supplementary methods in the event of failure of first-line management. Usually, repeated prolonged balloon inflation is performed at the site of or above the perforation with the lowest possible haemostatic pressure for 5-10 minutes until ischaemia or pain occurs, before measures enhancing coronary blood flow or cardiopulmonary resuscitation have to be implemented. If available, it is preferable to use a perfusion balloon catheter. Balloon use is not free of complications, as we have presented in our second case. As soon as the vessel rupture became evident in our patient, a balloon was placed just above the site of perforation to stop the bleeding. However, upon contrast injection, a pseudoaneurysm of the vessel wall at the site of balloon placement was seen. An injection of embolic material resolved the perforation and stopped blood flow into the pseudoaneurysm. Reversal of antithrombotic treatment is possible for a limited number of medications. Antidotes used in urgent situations, such as persistent contrast extravasation from a perforated artery, include protamine sulphate for unfractionated heparin, platelet transfusion for abciximab, and idarucizumab for dabigatran. Complete reversal is not possible for low-molecular-weight heparins, fondaparinux, or bivalirudin. GPIIb/ IIIa antagonists, eptifibatide, and tirofiban have no antidotes available. New antithrombotic agents are introduced successively, whereas antidotes are rarely developed later. Nevertheless, prognosis is related more to the type of perforation than to the used medications $[9,12,13]$. Peri-procedural medications could alter the course of otherwise self-limiting artery perforations. In our second case, a guidewire caused negligible perforation of the inferior thyroid artery because the bleeding ceased spontaneously. However, when the endovascular procedure was continued, the bleeding recurred, potentially due to compromised coagulation. Although a small perforation of a non-vital artery could seem clinically negligible compared to a more urgent emergency such as acute coronary syndrome (ACS), it can lead to serious complications if not managed. Farooqi et al. [14] described an exemplary guidewire-induced perforation of the costocervical trunk, which presented only after a coronary intervention. The complication remained unnoticed until a rapidly expanding cervical haematoma caused airway compromise leading to intubation and interventional therapy. Aortography revealed a pseudoaneurysm at the site of primary 
perforation. Since direct manual compression was not possible, an embolisation coil was placed in the feeder artery, and the bleeding resolved. The introduction of covered stents has successfully obviated the need to perform cardiac surgery in many cases of perforation, especially in large epicardial arteries involving proximal and middle segments [11]. There are reports of over $90 \%$ closure rates for polytetrafluoroethylene [PTFE]-covered stents for type I and II perforations; however, several criteria should be met to achieve such a closure rate - the target vessel must be accessible, of appropriate size, with no significant side branches, and the perforation easily seen $[6,11,15]$. There is a higher rate of subacute thrombosis with regard to covered stents in comparison to standard stents, probably because of delayed endothelialisation and compromised periprocedural anticoagulation therapy due to the perforation $[9,12,16]$. Coronary artery embolisation is the most radical non-surgical strategy for treating arterial perforation. Embolisation leads to permanent vessel closure beyond the site of deployment and possible subsequent infarction. The procedure itself requires experience with embolic materials, complex hardware, and manoeuvres that are not known to interventional cardiologists. Closure of one of the main coronary arteries might appear as a radical solution because coronary arteries are considered as terminal. In view of collateral circulation and instant haemodynamic compromise, permanent vessel closure seemed legitimate in our patient. In our case, extreme care had to be taken to use as little embolic material as possible in order not to close the first diagonal branch. Interventional radiologists have unique experience that is often needed when peripheral artery perforation occurs during a coronary intervention. We did not analyse perforations associated with arterial access, i.e. radial or femoral artery perforations, which have received substantial attention in the literature, and in turn we focused on the management of lesser-known perforations. Possible perforation sites, which may lead to morbid consequences, have been described in the literature. Overall, non-surgical management is successful in most cases of coronary perforation. Emergency coronary artery bypass surgery is required in $2.9 \%$ to $5.2 \%$ of cases $[3,4]$. Transcatheter treatment selection is related to the size of the artery, the volume of blood extravasation, and the parenchymal area at risk. The embolic materials and devices are selected based on their properties, vascular territory, and the desired endpoint of the therapeutic intervention. Vascular lesions that can be treated with selective embolisation include pseudoaneurysm, arteriovenous fistula, disruption of an arterial wall, and arterial perforation with contrast media extravasation. Embolisation is also indicated in cases of active intraparenchymal bleeding. We should answer several questions to successfully choose a proper embolisation method. First, embolisation should be specified as either proximal or peripheral. Proximal embolisation usually involves precise deployment of an embolic device such as a coil, plug, balloon, or stent. Peripheral perforation entails occlusion of precapillary vessels by a flowdirected agent, particles, or liquids. Available embolic materials can be divided into four categories: deployable devices, sponge elements, particulate agents, and liquid agents. Proximal occlusion immediately forms collateral circulation in most vascular territories, and it can be useful in the management of perforation of large arteries, as in our patient in whom the inferior thyroid artery was affected. Its function is to decrease blood pressure until haemostasis is achieved. Distal embolic agents occlude small precapillary structures and potentially lead to diffuse tissue necrosis. Another question is if we intend the embolisation to be permanent or temporary, and whether the site of perforation/bleeding requires precise deployment of a given device. Permanent occlusion can be achieved with the deployment of coils, which are most commonly used, and are available in a wide variety of shapes and sizes. Under certain circumstances, coils can be deployed quickly by injection. However, if we intend to achieve maximal precision, coils with a complex detachment mechanism should be required to avoid non-target vessel occlusion or an unsatisfactory position. Temporary embolisation can be achieved with a gelatine sponge a white, water-insoluble porous material that greatly facilitates clotting and thrombus development. Vascular occlusion also develops due to arteritis induced by foreign body placement. Within three weeks, the inflammatory reaction leads to vascular recanalisation. Its mechanism of action renders it mostly suitable for oncology and palliative treatment of bleeding, regardless of the site. Another available option involves the use of spherical polyvinyl alcohol (PVA) particles ranging in size from 100 to $1200 \mu \mathrm{m}$; this is a permanent embolic agent used solely for flow-directed precapillary occlusion. PVA particles penetrate deep into tissues, causing diffuse necrosis. This makes them most suitable for tumour embolisation. Lastly, liquid agents are the most challenging to manage and therefore their use requires special training. These agents include tissue adhesives (cyanoacrylate), sclerosants (absolute alcohol), and procoagulants (thrombin), which are often mixed with contrast agents to ensure opacification and slow polymerisation time. Cyanoacrylates are permanent agents used for occlusion of large and small vessels. Meticulous handling is of utmost importance since immediate polymerisation occurs after contact with blood or saline anions. Depending on the amount used, agents can cause blood flow redistribution or complete embolisation of the selected vessel. Ethanol causes immediate protein coagulation and clot formation. It is highly diffusible, and its use poses a risk of non-target vessel embolisation. Thrombin is used for treatment of pseudoaneurysms, either by percutaneous injection or transcatheter delivery to the feeding canal. In conclusion, artery perforation during cardiac catheterisation can lead to dire consequences. To manage this complication, clinicians need pre-established proce- 
dures, adequate resources, and knowledge. Interventional radiology can be considered as a salvage therapy in these cases.

\section{Conflict of interest}

The authors report no conflict of interest.

\section{References}

1. Witzke CF, Martin-Herrero F, Clarke SC, et al. The changing pattern of coronary perforation during percutaneous coronary intervention in the new device era. J Invasive Cardiol 2004; 16: 257-301.

2. Shimony A, Joseph L, Mottillo S, Eisenberg MJ. Coronary artery perforation during percutaneous coronary intervention: a systematic review and meta-analysis. Can J Cardiol 2011; 27: 843-850.

3. Fejka M, Dixon SR, Safian RD, et al. Diagnosis, management, and clinical outcome of cardiac tamponade complicating percutaneous coronary intervention. Am J Cardiol 2002; 90: 1183-1186.

4. Kiernan TJ, Yan BP, Ruggiero N, et al. Coronary artery perforations in the contemporary interventional era. J Interv Cardiol 2009; 22 350-353.

5. Kini AS, Rafael OC, Sarkar K, et al. Changing outcomes and treatment strategies for wire induced coronary perforations in the era of bivalirudin use. Catheter Cardiovasc Interv 2009; 74: 700-707.

6. Lansky AJ, Yang YM, Khan Y, et al. Treatment of coronary artery perforations complicating percutaneous coronary intervention with a polytetrafluoroethylene-covered stent graft. Am J Cardiol 2006; 98: 370-374.

7. Lee SY, Kim SM, Bae JW, et al. Renal artery perforation related with hydrophilic guide wire during coronary intervention: successful treatment with polyvinyl alcohol injection. Can J Cardiol 2012; 28 : 612.e5-7.

8. Patel T, Shah S, Kuladhipati I. Management of guidewire-induced coronary artery perforations through transradial route - a simple approach. J Invasive Cardiol 2009; 21: E248-251.
9. Dippel EJ, Kereiakes DJ, Tramuta DA, et al. Coronary perforation during percutaneous coronary intervention in the era of abciximab platelet glycoprotein IIb/IIIa blockade: an algorithm for percutaneous management. Catheter Cardiovasc Interv 2001; 52: 279-286.

10. Ellis SG, Ajluni S, Arnold AZ, et al. Increased coronary perforation in the new device era. Incidence, classification, management, and outcome. Circulation 1994; 90: 2725-2730.

11. Jamshidi P, Mahmoody K, Erne P. Covered stents: a review. Int J Cardiol 2008; 130: 310-318.

12. Gomez-Outes A, Suarez-Gea ML, Lecumberri R, et al. Specific antidotes in development for reversal of novel anticoagulants: a review. Recent Pat Cardiovasc Drug Discov 2014; 9: 2-10.

13. Levi M. Emergency Reversal Strategies for Anticoagulation and Platelet Disorders. Front Neurol Neurosci 2015; 37: 51-61.

14. Farooqi F, Alexander J, Sarma A. Rare vascular perforation complicating radial approach to percutaneous coronary angioplasty. BMJ Case Rep 2013; 2013: doi: 10.1136/bcr-2012-007732.

15. Briguori C, Nishida T, Anzuini A, et al. Emergency polytetrafluoroethylene-covered stent implantation to treat coronary ruptures. Circulation 2000; 102: 3028-3031.

16. Takano M, Yamamoto M, Inami S, et al. Delayed endothelialization after polytetrafluoroethylene-covered stent implantation for coronary aneurysm. Circ J 2009; 73: 190-193. 